\title{
Surgical approaches to apical thoracic malignancies
}

\author{
Marc de Perrot, MD, MSc, ${ }^{a}$ and Raja Rampersaud, MD $^{\mathrm{b}}$
}

Objective: Several surgical approaches have been described to access apical thoracic malignancies extending into the thoracic inlet. However, most publications have focused on a specific approach and considered the thoracic inlet as 1 entity. In the present analysis, we divided the thoracic inlet into 5 different zones requiring specific surgical considerations to identify the best approach for each zone.

Methods: A review of 22 consecutive patients undergoing surgery for apical thoracic malignancies extending into the thoracic inlet from January 2005 to November 2011 was performed.

Results: Different surgical approaches were used for each zone. The first (anterolateral) zone required a subclavicular approach to open the costoclavicular space and expose the subclavian vein with or without elevating or removing the clavicle $(n=4)$. The second (anterocentral) zone required a transverse supraclavicular approach with or without extension to a partial (trapdoor) or full sternotomy $(\mathrm{n}=10)$. The third (posterosuperior) zone located between the top of the subclavian artery and the T1 vertebra along the posterior superior border of the first rib was the most difficult area to access $(\mathrm{n}=5)$. The transclavicular approach was ideally suited to expose this zone in our experience. The fourth (posteroinferior) zone and fifth (inferolateral) zone located posteriorly and laterally along the inferior border of the first rib were accessed using a posterolateral and posterotransaxillary approach, respectively $(\mathrm{n}=3)$.

Conclusions: The thoracic inlet could be divided into 5 zones requiring specific surgical considerations and different approaches. Division of the thoracic inlet into these zones could provide more clarity and guidance for thoracic surgeons to select the correct surgical approach. (J Thorac Cardiovasc Surg 2012;144:72-80)

The surgical approach to apical thoracic malignancies represents a technical challenge owing to the important neurovascular structures confined in the limited space between the first rib, manubrium, and first thoracic vertebra (T1). The thoracic inlet is classically divided around the subclavian artery into anterior, middle, and posterior compartments, with an anterior approach typically used to access the anterior and middle compartment and a posterior approach to access the posterior compartment. ${ }^{1,2}$ A number of anterior and posterior approaches have been described to achieve the best exposure with the lowest morbidity. Each of these approaches offers specific advantages and drawbacks and should therefore be selected according to the type and locoregional extension of the tumor. ${ }^{3}$ However, it is often difficult to choose the optimal approach for a specific tumor, because most publications have focused on a specific approach and considered the thoracic inlet as 1 entity. In the present study, we divided the thoracic inlet into 5 different zones requiring specific surgical considerations and different surgical approaches.

From the Division of Thoracic Surgery ${ }^{\mathrm{a}}$ and Division of Orthopedic Surgery, ${ }^{\mathrm{b}}$ University Health Network, University of Toronto, Toronto, Ontario, Canada.

Disclosures: Authors have nothing to disclose with regard to commercial support.

Received for publication Jan 17, 2012; revisions received March 14, 2012; accepted

for publication March 19, 2012; available ahead of print April 16, 2012.

Address for reprints: Marc de Perrot, MD, MSc, Division of Thoracic Surgery, Tor-

onto General Hospital, 9N-961, 200 Elizabeth St, Toronto, ON M5G 2C4, Canada

(E-mail: marc.deperrot@uhn.on.ca).

$0022-5223 / \$ 36.00$

Copyright (C) 2012 by The American Association for Thoracic Surgery doi: $10.1016 /$ j.jtcvs.2012.03.049

\section{METHODS}

A retrospective review of a prospective database including 984 patients from a single surgeon (M.d.P.) identified 22 consecutive patients undergoing resection of malignant tumors at the level of the thoracic inlet from January 2005 to November 2011 (Figure 1). The institutional review board approved the study, and informed consent was waived.

\section{Patients}

Most patients $(\mathrm{n}=10)$ presented with non-small cell lung cancer (NSCLC), 8 presented with metastatic disease and 4 with a mediastinal mass extending to the thoracic inlet (Table 1). All patients underwent disease staging with computed tomography of the chest and abdomen. Patients with NSCLC also underwent mediastinoscopy, brain magnetic resonance imaging, and bone scanning and/or positron emission tomography. Magnetic resonance imaging of the spine and thoracic inlet was performed for tumors extending along the pulmonary sulcus. The clinical stage of patients with NSCLC was cT3NO in 5 and cT4N0 in 5. All but 2 patients with NSCLC had undergone induction chemoradiotherapy, with a radiation dose of 30 to $66 \mathrm{~Gy}$ (median, $45 \mathrm{~Gy}$ ), and 9 of 12 patients with metastatic disease or mediastinal mass had induction chemotherapy before surgery. ${ }^{4}$ All patients with NSCLC underwent en bloc resection of the tumor with lobectomy $(\mathrm{n}=8)$ or pneumonectomy $(\mathrm{n}=2)$. The anatomic structures resected en bloc with the tumor were the chest wall in 8 , vertebra in 6 , subclavian vein in 6, sympathetic trunk in 4 , T1 nerve root in 3, phrenic nerve in 3, subclavian artery in 2, vertebral artery in 1, vagus nerve in 1, and C8-T1 nerve roots, together with the inferior trunk of the brachial plexus in 1.

\section{Zones}

A schematic representation of the 5 zones is presented in Figure 2. The first zone (anterolateral zone) is located anteriorly and laterally to the subclavian vein between the lateral border of the sternum and the anterior scalene muscle. This zone includes the anterior part of the first rib and the subclavian vein. Access to this zone requires opening the costoclavicular space to expose the subclavian vein. The second zone (anterocentral 


\section{Abbreviation and Acronym \\ NSCLC $=$ non-small cell lung cancer}

zone) is located medially to the subclavian vein and contains the subclavian artery up to the middle part of the first rib. The tumors included in this zone typically extend along the subclavian artery and/or brachiocephalic vein and can be accessed without opening the costoclavicular space. The third zone (posterosuperior zone) extends from the top of the subclavian artery to the $\mathrm{T} 1$ vertebra and encompasses the posterior superior border of the first rib. The tumors located in this zone typically extend along the vertebral artery and/or involve the T1 vertebra at the level of the costovertebral articulation, neural foramen, and/or vertebral body. Because of the cephalad course of the first rib, structures located at the level of T1 and immediately anterior to T1 sit outside the chest and thus cannot be adequately accessed with a posterior approach alone. The fourth zone (posteroinferior zone) and fifth zone (inferolateral zone) extend posteriorly and laterally along the inferior border of the first rib. The fourth zone extends medially to the spine, and the fifth zone extends laterally to the axilla and subclavian artery after it exits the window between the anterior and middle scalene muscles. Tumors located in zone 4 and/or zone 5 do not involve the T1 costovertebral articulation, T1 neural foramen, and/or T1 vertebral body.

\section{Surgical Approach}

The surgical approach can be selected according to the locoregional extension of the tumor in these different zones of the thoracic inlet (Table 2). These approaches have been previously described in published studies ${ }^{5-16}$; therefore, the surgical details were not included in the present report.

Tumors located in zone 1 are accessed using a subclavicular approach with or without a vertical cervicotomy along the sternocleidomastoid muscle. The subclavicular approach is performed by an incision along the upper edge of the second rib below the clavicle, providing exposure to the first rib after dividing the pectoralis major muscle along its fibers and retracting the pectoralis minor muscle laterally. ${ }^{8}$ Sectioning of the costochondral junction of the first rib and division of the costoclavicular ligament opens the costoclavicular space and provides access to the anterior part of the first rib up to the subclavian vein and anterior scalene muscle. The subclavicular incision can be combined with a vertical cervicotomy along the anterior border of the sternocleidomastoid muscle in an L-shape fashion to either split the manubrium and elevate the sternoclavicular articulation with the clavicle (transmanubrial approach) or divide the sternal attachment of the sternocleidomastoid muscle and remove the proximal part of the clavicle (transcervical-thoracic approach) to have adequate exposure of the subclavian vein and artery. ${ }^{10,13}$

Tumors located in zone 2 are accessed by a transverse supraclavicular approach. This approach is performed by a transverse cervical skin incision $2 \mathrm{~cm}$ above the clavicle. ${ }^{5}$ After dissection of the prescalenic lymph nodes and identification of the phrenic nerve, the anterior scalene muscle can be sectioned to expose the subclavian artery. The transverse supraclavicular approach can be combined with a full sternotomy or partial sternotomy extending into the third or fourth intercostal space (trapdoor approach) according to the intrathoracic extension of the tumor. ${ }^{6,7}$ The supraclavicular approach provides good exposure to the subclavian artery and brachiocephalic vein but very limited exposure to the subclavian vein. ${ }^{6}$ If the subclavian vein is involved, the costoclavicular space needs to be opened as described in zone 1 , with resection of the first rib or clavicle. ${ }^{15,17-19}$

Tumors located in zone 3 are accessed by a vertical cervicotomy along the anterior border of the sternocleidomastoid muscle extending into the upper part of the sternum. The sternocleiodmastoid muscle is then reflected laterally by splitting the upper part of the sternum down to the first intercostal space (cervical-transsternal approach) or below (hemiclamshell approach). ${ }^{11,12}$ Division of the sternocleidomastoid muscle at its lower insertion sites, leaving adequate length to reattach it at the end of the operation, is occasionally required to provide additional access to the posterosuperior zone. The transclavicular approach provides optimal exposure to the posterosuperior zone by extending the vertical cervicotomy down to the upper border of the third rib, sectioning the sternocleidomastoid and pectoralis major muscles off the chest wall to create a myocutaneous flap that is reflected laterally and resecting the proximal part of the clavicle., ${ }^{9,20,21}$ This approach provides complete reflection of the sternocleidomastoid muscle to the posterior triangle of the neck and gives optimal anterolateral access to the spine from $\mathrm{C} 7$ to T4. The first and second ribs are exposed without opening the sternum, and the lung is approached through an anterior thoracotomy in the second intercostal space.

The posterior approach to the thoracic inlet requires an incision extending posteriorly between the scapula and the spine up to the $\mathrm{C} 7$ vertebra, with division of the latissimus dorsi muscle, trapezius, levator scapulae, and rhomboid major and minor muscles. This extended posterior incision is commonly used to provide exposure to zone 4 of the thoracic inlet. ${ }^{14}$ The posterotransaxillary approach requires the same extended posterior incision to the $\mathrm{C} 7$ vertebra, but the anterior part of the incision is curved along the scapula into the axilla by dividing the serratus anterior muscle up to the pectoralis major muscle in a hook fashion, as described by Niwa and colleagues ${ }^{15}$ and Tatsumura and colleagues. ${ }^{16}$ The transaxillary extension of the posterior approach improves the lateral exposure to the subclavian vessels in zone 5 .

\section{RESULTS}

Of 22 patients with thoracic malignancies extending to the thoracic inlet, 4 were located in zone 1,10 in zone 2 , 5 in zone 3, 2 in zone 4, and 1 in zone 5 (Figure 1). A representative computed tomography scan of NSCLC located in each zone is presented in Figure 3.

Tumors located in zone 1 were accessed with a subclavicular approach (Figure 4). If the subclavian vessels were not involved by the tumor, the subclavicular approach was adequate to expose the anterior part of the first rib, dissect the subclavian vein, and section the first rib between the sternum and anterior scalene muscle without elevating or resecting the clavicle $(\mathrm{n}=2)$. When the subclavian vessels were involved, the subclavicular incision was combined with a vertical cervical incision along the sternocleidomastoid muscle to elevate $(\mathrm{n}=1)$ or remove $(\mathrm{n}=1)$ the clavicle. The subclavicular approach did not provide access to the pulmonary hilum; thus, both patients with NSCLC underwent an anatomic lung resection through a planned separate anterior thoracotomy in the same operative setting.

Tumors located in zone 2 were approached through a supraclavicular incision (Figure 4). The supraclavicular exposure was combined with partial sternotomy $(\mathrm{n}=3)$ or full sternotomy $(\mathrm{n}=2)$ to complete the intrathoracic resection of the tumor. This incision provided excellent exposure for tumors extending medially along the subclavian artery and/or brachiocephalic vein. The subclavian vein, however, was not involved in this group of patients. Both patients with NSCLC underwent an anatomic lung resection through the trapdoor incision. 


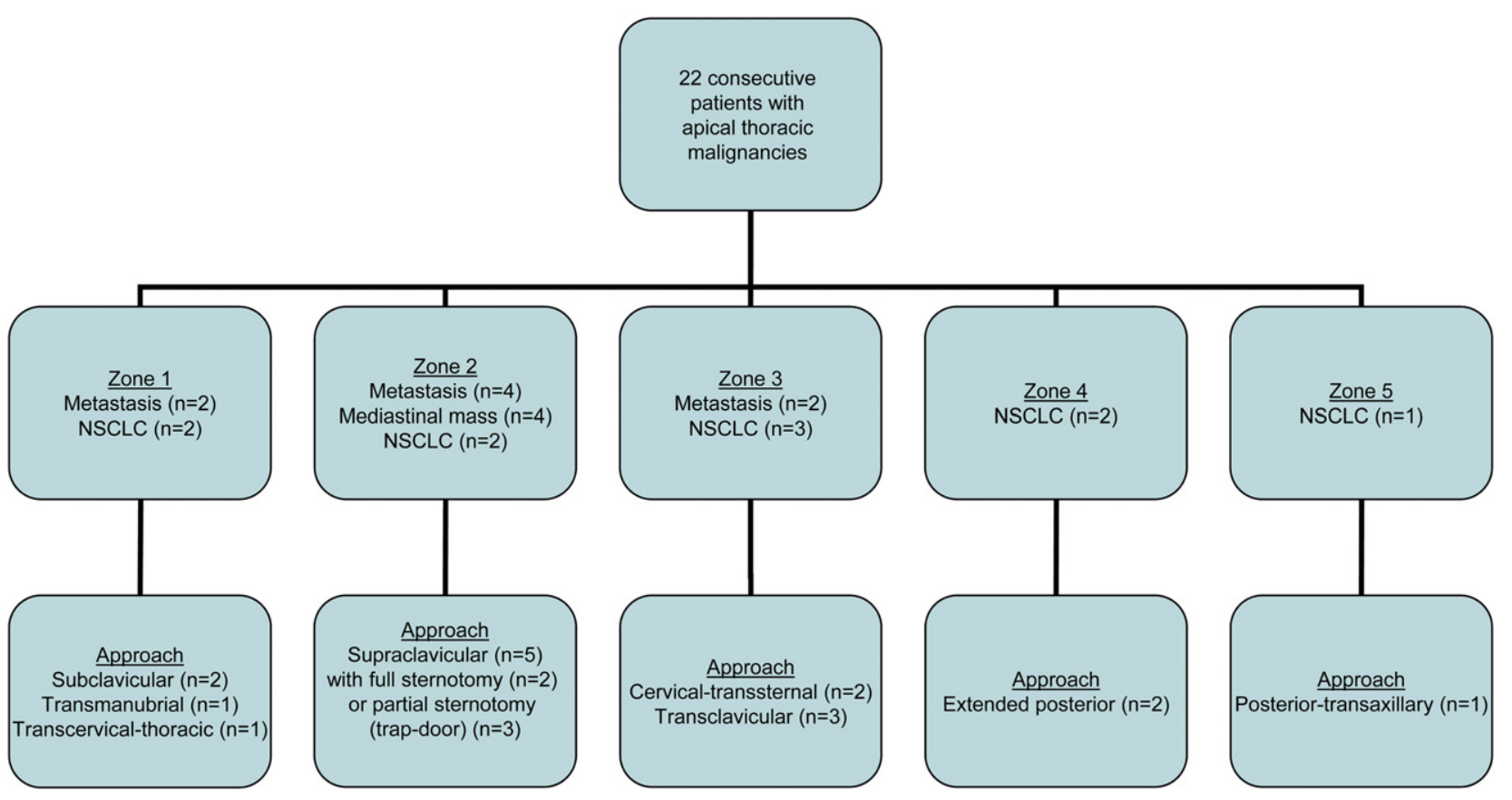

FIGURE 1. Distribution of patients and surgical approaches by zone. NSCLC, Non-small cell lung cancer.

In both patients with metastatic disease located in zone 3 , the vertical cervicotomy along the sternocleidomastoid muscle with partial sternotomy ending in the first intercostal space provided good exposure to the vertebral body of $\mathrm{T} 1$ and T2 without removing the clavicle (Figure 4). In patients with NSCLC located in zone 3, a transclavicular approach was required to adequately expose this zone and be able to remove the tumor en bloc with an anatomic lung resection (Figure 4). The lung resection was performed through an anterior thoracotomy in the second intercostal space. These patients presented with involvement of the T1

TABLE 1. Patient and tumor characteristics

\begin{tabular}{lc}
\hline \multicolumn{1}{c}{ Characteristic } & Value \\
\hline Age (y) & $48 \pm 18$ \\
Gender (n) & \\
Male & 17 \\
Female & 5 \\
Side of surgery (n) & \\
Left & 16 \\
Right & 6 \\
NSCLC (n) & 10 \\
Metastatic disease (n) & 8 \\
Testicular germ cell tumor & 4 \\
Sarcoma & 2 \\
Renal cell carcinoma & 1 \\
Breast carcinoma & 1 \\
Mediastinal mass (n) & 4 \\
Primary mediastinal germ cell tumor & 3 \\
Thymoma & 1 \\
\hline
\end{tabular}

NSCLC, Non-small cell lung cancer. vertebra $(n=3)$ and/or vertebral artery $(n=1)$. The transclavicular approach was performed after a posterior midline approach to section the nerve roots in the neural foramen $(\mathrm{n}=1)$ or spinal canal $(\mathrm{n}=2)$ and to stabilize the spine posteriorly for patients requiring hemivertebrectomy $(\mathrm{n}=1)$ or total vertebrectomy of T1 to T3 $(\mathrm{n}=1)$. This initial posterior spinal and paraspinal soft tissue release, as well as spinal osteotomies and instrumentation, allowed us to complete the en bloc resection of the tumor in 1 setting through the anterior transclavicular approach. An anterior spinal stabilization was also performed through the same approach in 1 patient who underwent total vertebrectomy of $\mathrm{T} 1$ to $\mathrm{T} 3$ en bloc with the right upper lobectomy (Figure 5).

Tumors located in zone 4 or 5 extended posteriorly and laterally along the inferior border of the first rib and did not involve the $\mathrm{T} 1$ costovertebral articulation, $\mathrm{T} 1$ neural foramen, and/or T1 vertebral body. Both tumors located in zone 4 were approached through an extended posterolateral incision and required resection of the transverse process of $\mathrm{T} 1$ to expose T1 nerve root with partial $(\mathrm{n}=1)$ or total $(\mathrm{n}=1)$ vertebrectomy of T2 to T4 (Figure 4). Because the T1 vertebra was not involved, the spinal osteotomies and instrumentation of $\mathrm{T} 2$ to $\mathrm{T} 4$ were performed by combining the extended posterior approach with a posterior midline incision without any anterior cervical approach. Tumor located in zone 5 was accessed through a posterotransaxillary approach. This approach provided excellent exposure to the subclavian vessels distally to the lateral border of the anterior and middle scalene muscles (Figure 6). 


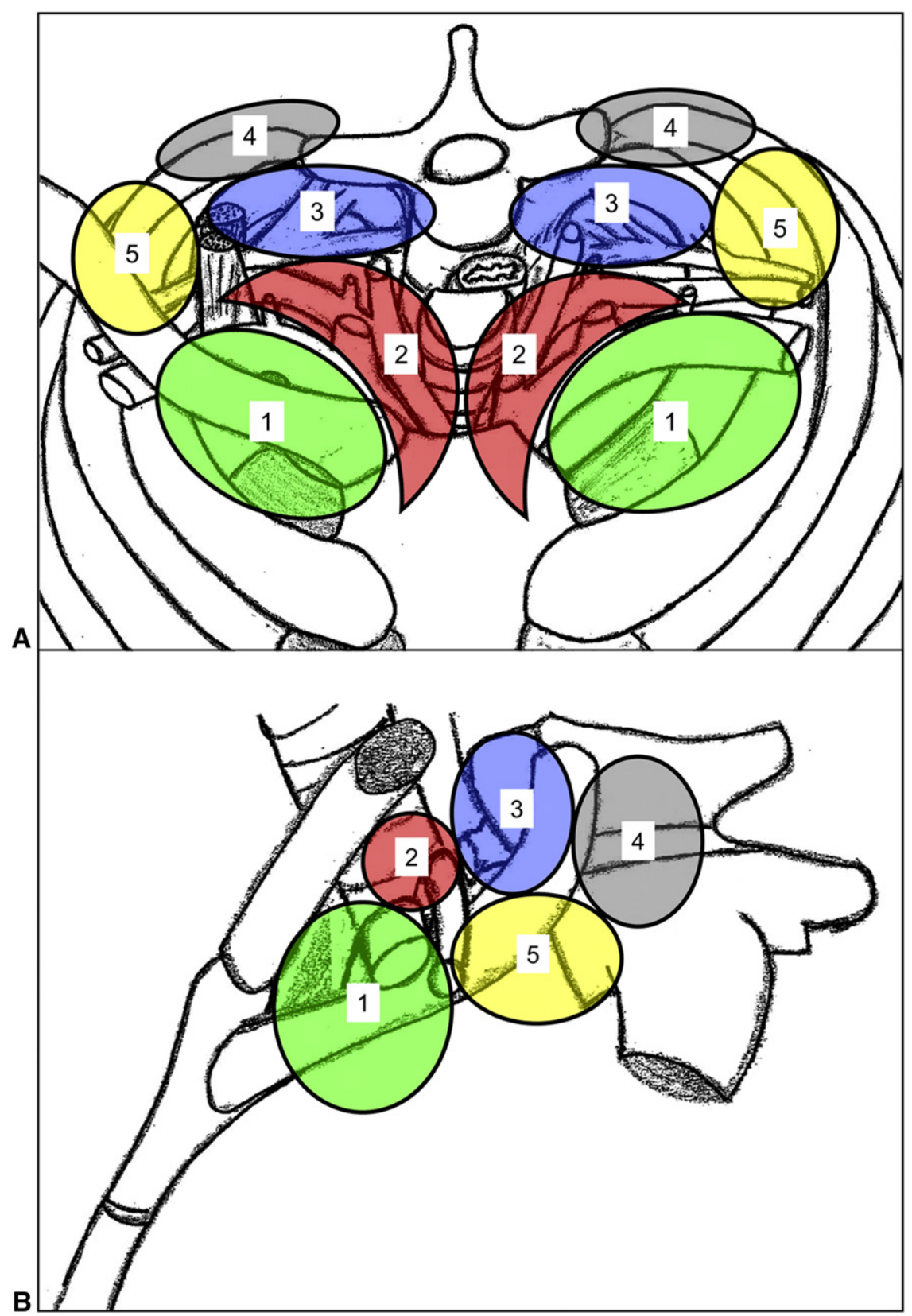

FIGURE 2. Superior (A) and lateral (B) view of thoracic inlet. The green zone (zone 1) represents the anterolateral zone located between the sternum and anterior scalene muscle and requiring exposure of the subclavian vein in the costoclavicular space. The red zone (zone 2) represents the anterocentral zone containing the subclavian artery medially to the first rib. The blue zone (zone 3) represents the posterosuperior zone located between the top of the subclavian artery and T1 vertebra, encompassing the posterior superior border of the first rib and the vertebral artery. The gray zone (zone 4) represents the posteroinferior zone located along the posterior inferior border of the first rib extending toward the spine. The yellow zone (zone 5) represents the inferolateral zone located along the posterior inferior border of the first rib and extending toward the subclavian artery distally to the anterior and middle scalene muscles.

With the exception of the 2 patients undergoing spinal decompression for metastatic disease in zone 3, all but 2 of the remaining patients underwent $\mathrm{R} 0$ resection $(90 \%)$. One patient with NSCLC located in zone 2 underwent R1 resection along the dome of the aortic arch, and 1 patient with metastatic disease located in zone 2 underwent R2 resection along the carotid artery that was not resected and reconstructed owing to the age and comorbidities of the patient. The final pathologic stage of the patients undergoing surgery for NSCLC was pT3N0 in 1, pT4N0 in 1, ypT0N0 in 1, ypT3N0 in 2, ypT3N1 in 2, ypT4N0 in 1, ypT4N1 in 1, and ypT4N3 in 1. One patient with NSCLC died 28 days after undergoing left pneumonectomy of pneumonia, for an overall 30 -day operative mortality of $4.5 \%$. 
TABLE 2. Surgical approaches

\begin{tabular}{|c|c|c|}
\hline Approach* (original investigator) & Exposure & Comments \\
\hline Subclavicular (Murphy et $\mathrm{al}^{8}$ ) & Zone 1 & Exposes subclavian vein, but no exposure of subclavian artery \\
\hline Transmanubrial (Grunenwald et $\mathrm{al}^{10}$ ) & Zone 1 and 2 & Good exposure of subclavian vessels \\
\hline Transcervical-thoracic (Dartevelle et al $^{13}$ ) & Zone 1-3 & Resection of clavicle provides excellent exposure of subclavian vessels \\
\hline Transverse supraclavicular (Blanchereau et $\mathrm{al}^{5}$ ) & Zone 2 & Exposes subclavian artery but limited exposure of subclavian vein \\
\hline $\begin{array}{l}\text { Transverse supraclavicular with sternotomy/trapdoor } \\
\left(\text { Hajarizadeh et } \mathrm{al}^{6} ; \text { Masaoka et } \mathrm{al}^{7} \text { ) }\right.\end{array}$ & Zone 2 & Resection of clavicle or first rib required to access zone $1^{15,17-19}$ \\
\hline $\begin{array}{l}\text { Cervical-transsternal/hemiclamshell } \\
\left(\text { Ladas et } \mathrm{al}^{11} ; \text { Korst et } \mathrm{al}^{12} \text { ) }\right.\end{array}$ & Zone 2 and 3 & Section of sternocleiodmastoid muscle required for large tumor in zone 3 \\
\hline Transclavicular (de Perrot ${ }^{9}$; Fadel et $\mathrm{al}^{20}$ ) & Zone 1,2 and 3 & Anterolateral access to spine from $\mathrm{C} 7$ to $\mathrm{T} 4$ \\
\hline Extended posterior (Shaw et $\mathrm{al}^{14}$ ) & Zone 4 & Limited access to zone 3 \\
\hline $\begin{array}{l}\text { Posterior-transaxillary/hook } \\
\left(\text { Niwa et } \mathrm{al}^{15} \text {; Tatsumura et } \mathrm{al}^{16}\right)\end{array}$ & Zone 4 and 5 & Good exposure of subclavian vessels in axilla \\
\hline
\end{tabular}

\section{DISCUSSION}

Division of the thoracic inlet into 5 zones can facilitate the selection of the most appropriate surgical approach, because each of these 5 zones requires specific surgical considerations. Zone 1 requires exposure of the costoclavicular space to dissect the subclavian vein. The tumors located in this zone were accessed by a subclavicular approach with or without elevation or resection of the clavicle. Tumors located in zone 2 are in close proximity to the subclavian artery and/or brachiocephalic vein but do not involve the subclavian vein and thus could be accessed through a supraclavicular approach associated with a partial hemiclamshell sternotomy (trapdoor) or full sternotomy according to the intrathoracic extension of the tumor.
Tumors located in zone 3 are the most difficult to access, because they are located between the top of the subclavian artery and $\mathrm{T} 1$ vertebra and are in close proximity to the brachial plexus and vertebral artery. Because of the cephalad course of the first rib, this zone is located outside the chest and must be approached anteriorly by an incision extending down the sternocleidomastoid muscle to the upper part of the sternum. The cervical component of the incision should extend high in the neck to facilitate the lateral retraction of the sternocleidomastoid muscle and improve exposure to the thoracic inlet. Although a partial upper sternotomy provides lateral retraction of the sternocleidomastoid muscle, the exposure to zone 3 remains limited unless the sternocleidomastoid is sectioned at its lower insertion on the sternum
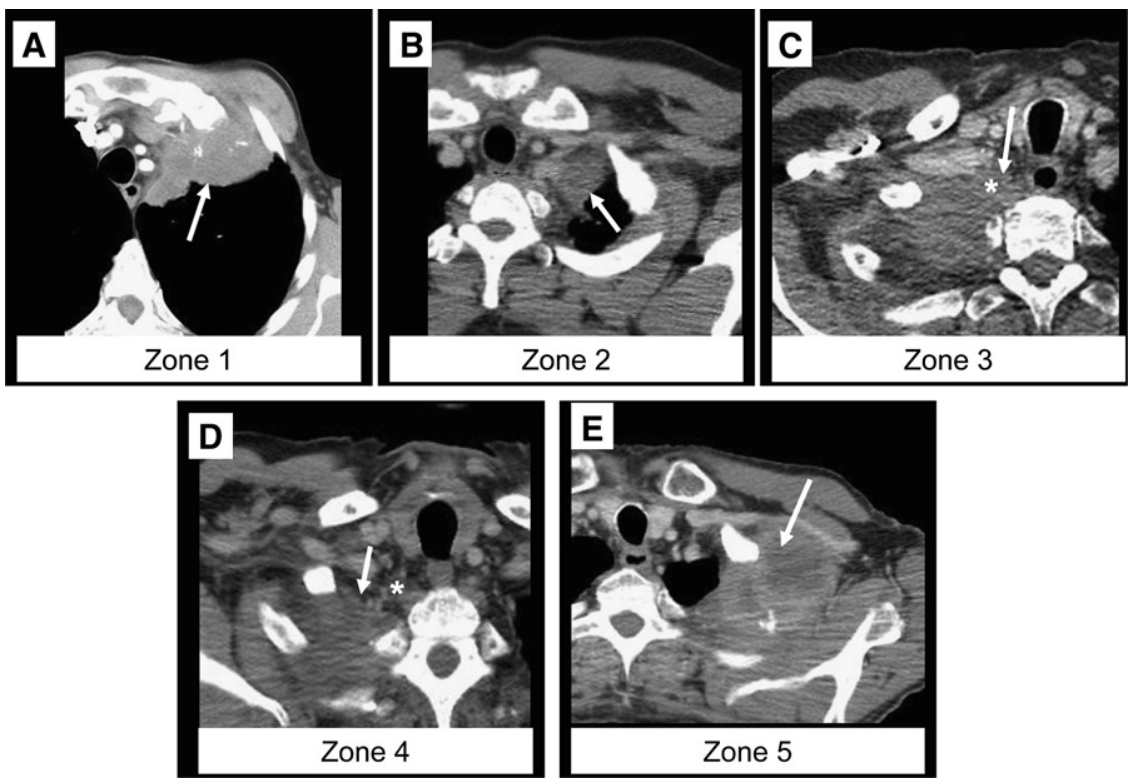

FIGURE 3. Computed tomography scan of the chest demonstrating the location of tumor in the different zones of the thoracic inlet in patients with nonsmall cell lung cancer (white arrows). A, Tumor located in zone 1. B, Tumor located in zone 2. C, Tumor located in zone 3 between the top of the subclavian artery and the $\mathrm{T} 1$ vertebra. D, Tumor located in zone 4 . E, Tumor located in zone 5. Note the mediastinal tissue between the subclavian artery and T1 (asterisk) is not involved in tumor located in zone 4 (D) compared to the tumor located in zone 5 (C). 


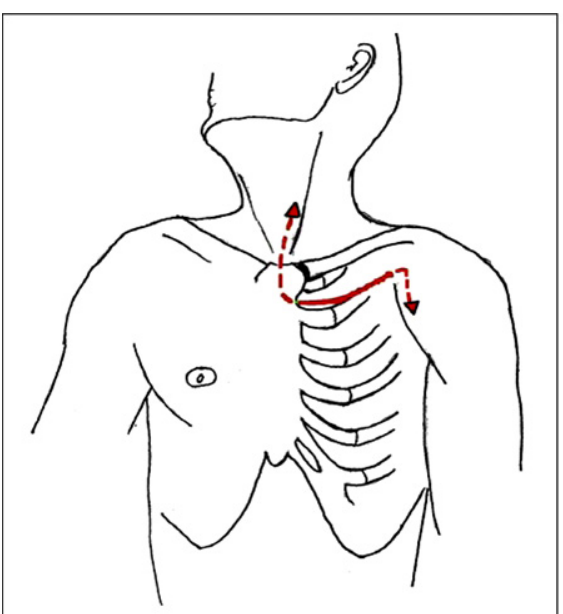

A Zone 1

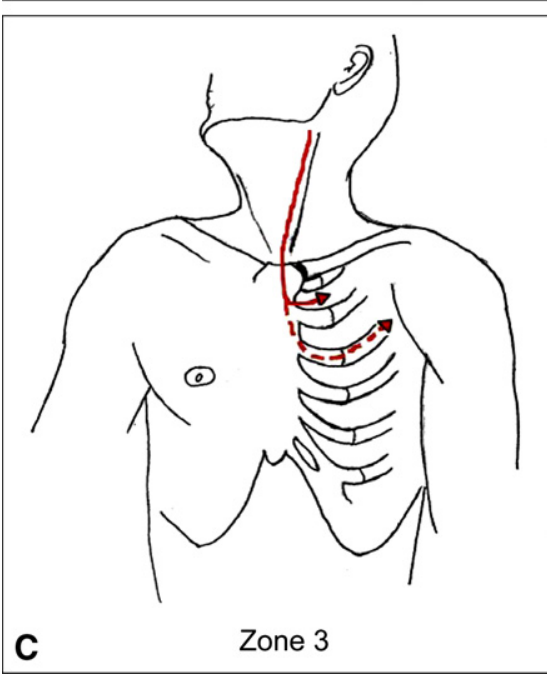

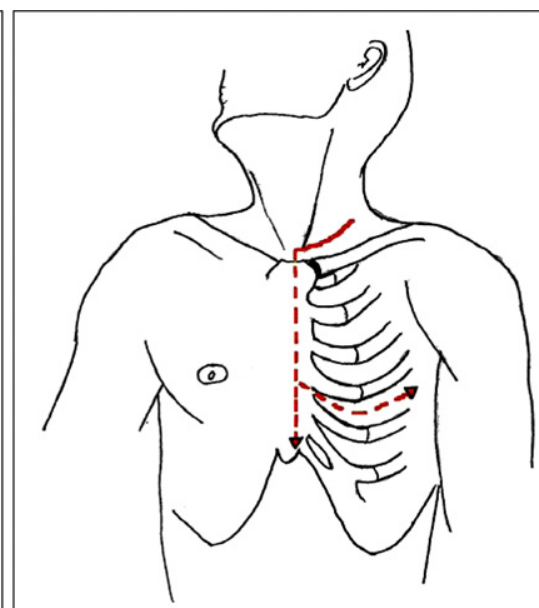

Zone 2

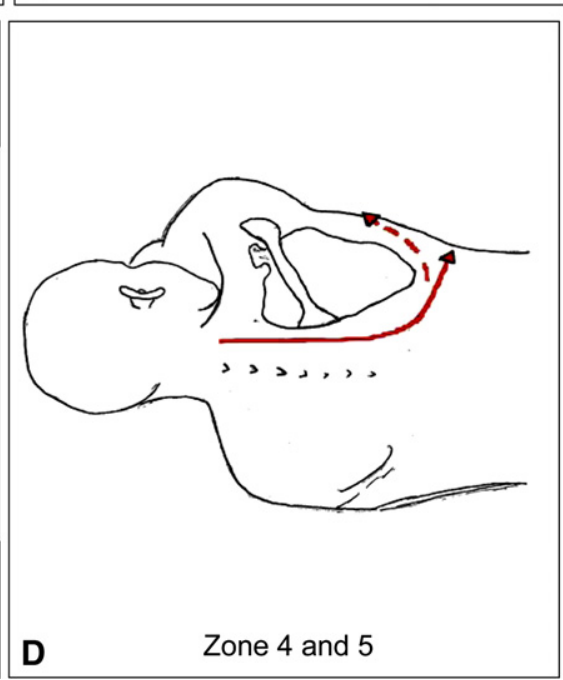

FIGURE 4. A, Zone 1 required exposure of the subclavian vein in the costoclavicular space and was approached through a subclavicular incision ( red line). The incision was extended along the sternocleiodmastoid muscle to elevate or resect the clavicle if the subclavian vein and artery needed to be exposed (dashed line). The incision could also be extended into the deltopectoral groove to access the subclavian vessels beyond the clavicle, if necessary (dashed line). This approach was associated with a separate anterior thoracotomy to perform the anatomic lung resection in patients with non-small cell lung cancer. $\mathrm{B}$, Zone 2 was approached through a supraclavicular incision (red line). The incision was associated with a partial or full sternotomy (dashed line), according to the intrathoracic extension of the tumor. This incision provided good exposure of the subclavian artery and brachiocephalic vein but very limited exposure of the subclavian vein. C, Zone 3 was approached through an incision along the sternocleidomastoid muscle extending into the first intercostal space (red line). After splitting the manubrium, this incision provided good exposure of the T1 and $\mathrm{T} 2$ vertebral body but limited exposure of the posterior part of the first rib. Therefore, a transclavicular approach with extension of the incision into the second intercostal space associated with mobilization of the sternocleidomastoid and major pectoralis muscles as a myocutaneous flap combined with resection of the proximal part of the clavicle was used for patients with NSCLC located in zone 3 (dashed line). Resection of the inner part of the clavicle and mobilization of the sternocleidomastoid muscle to the posterior triangle of the neck provided excellent anterolateral exposure to the posterosuperior zone and spine between $\mathrm{C} 7$ and $\mathrm{T} 4$. The anatomic lung resection could be performed through the anterior thoracotomy in the second intercostal space. D, Zone 4 was approached through a posterior incision extending up to the $\mathrm{C} 7$ vertebra (red line). In tumors extending to the subclavian vessels in zone 5, the incision was extended around the scapula to the lateral border of the major pectoralis muscle in the axilla (dashed line).

and clavicle. In our experience, the transclavicular approach was ideally suited to expose zone 3 by completely mobilizing the sternocleidomastoid muscle to the posterior triangle of the neck and providing excellent anterolateral exposure to the spine between the $\mathrm{C} 7$ and $\mathrm{T} 4$ vertebra. ${ }^{9,20}$ Total vertebrectomy of T1 to T3 for NSCLC, en bloc with right upper lobectomy, followed by anterior spinal stabilization (typically with first stage posterior release and spinal stabilization) could be achieved with this approach.

Resection of the proximal part of the clavicle and upper chest wall does result in some cosmetic and functional impairment of the shoulder, although less pronounced if 

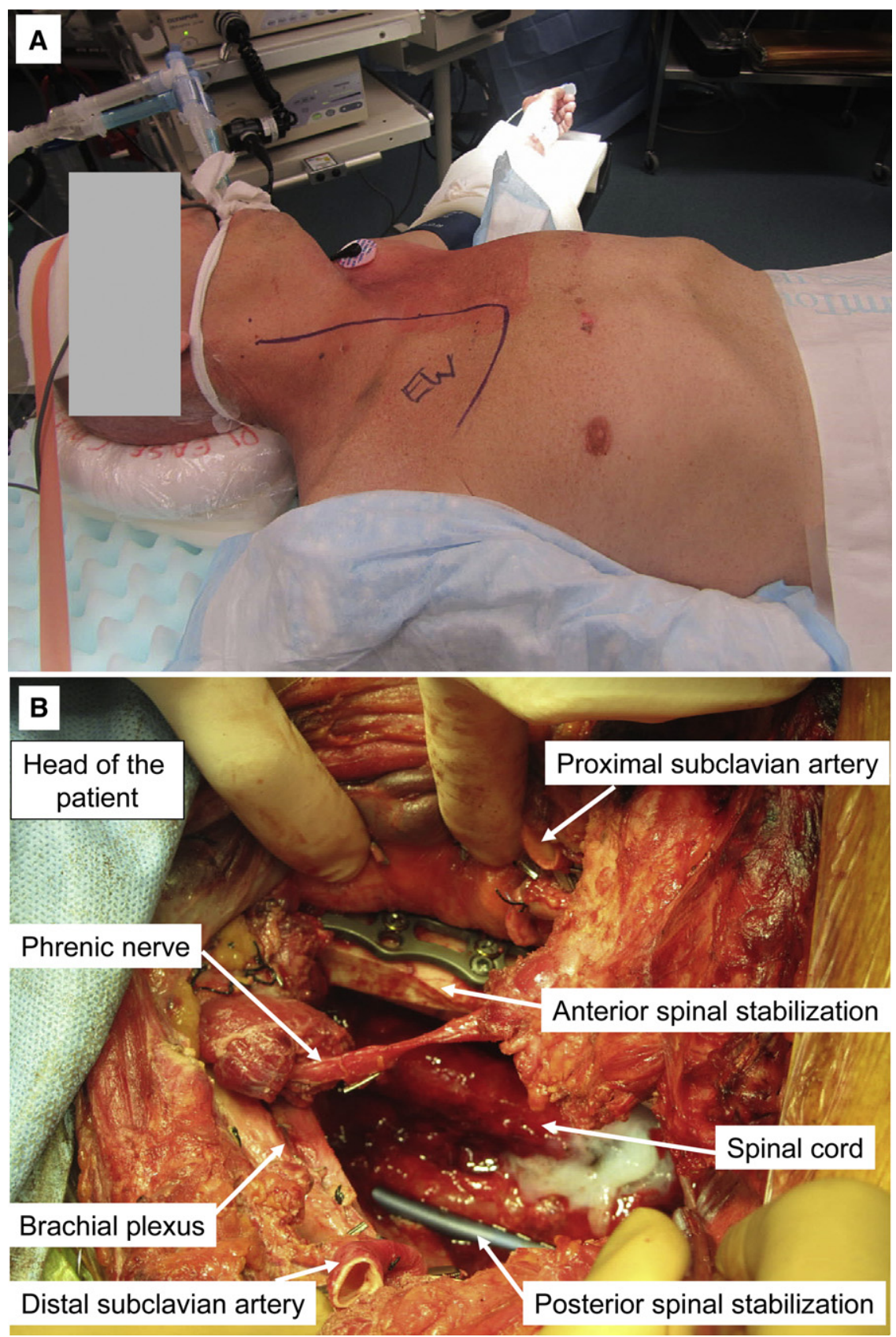

FIGURE 5. Intraoperative view of a patient undergoing total vertebrectomy of $\mathrm{T} 1$ to $\mathrm{T} 3 \mathrm{en}$ bloc, with ribs 1 to 4 , subclavian vessels, and the right upper lobe for non-small cell lung cancer. A, The transclavicular approach extending along the sternocleidomastoid muscle down to the second intercostal space was performed 10 days after a posterior midline approach for laminectomy and posterior spinal stabilization. B, This approach allowed complete en bloc resection of the tumor and anterior stabilization of the spine through the same incision. A and B, The same view from the right side of the patient.

a posterolateral thoracotomy is avoided (as is our practice) and the scapulothoracic articulation is preserved. ${ }^{21}$ The degree of dysfunction also varies according to the extent of upper chest wall resection and becomes more prominent in patients requiring resection of the first 3 or 4 ribs. Some investigators have reported different techniques to preserve and reconstruct the clavicle. ${ }^{22,23}$ Preservation of the anterior part of the clavicle on a pedicle of sternocleidomastoid muscle was most recently described; however, the risk of malunion is high when the clavicle is reconstructed after radiotherapy. ${ }^{23,24}$

The posterior approach with a large posterolateral incision extending up to $\mathrm{C} 7$ as described by Shaw and colleagues ${ }^{14}$ was used for posterior tumors extending to the inferior border of the first rib. The posterior incision was extended around the scapula up to the lateral border of 

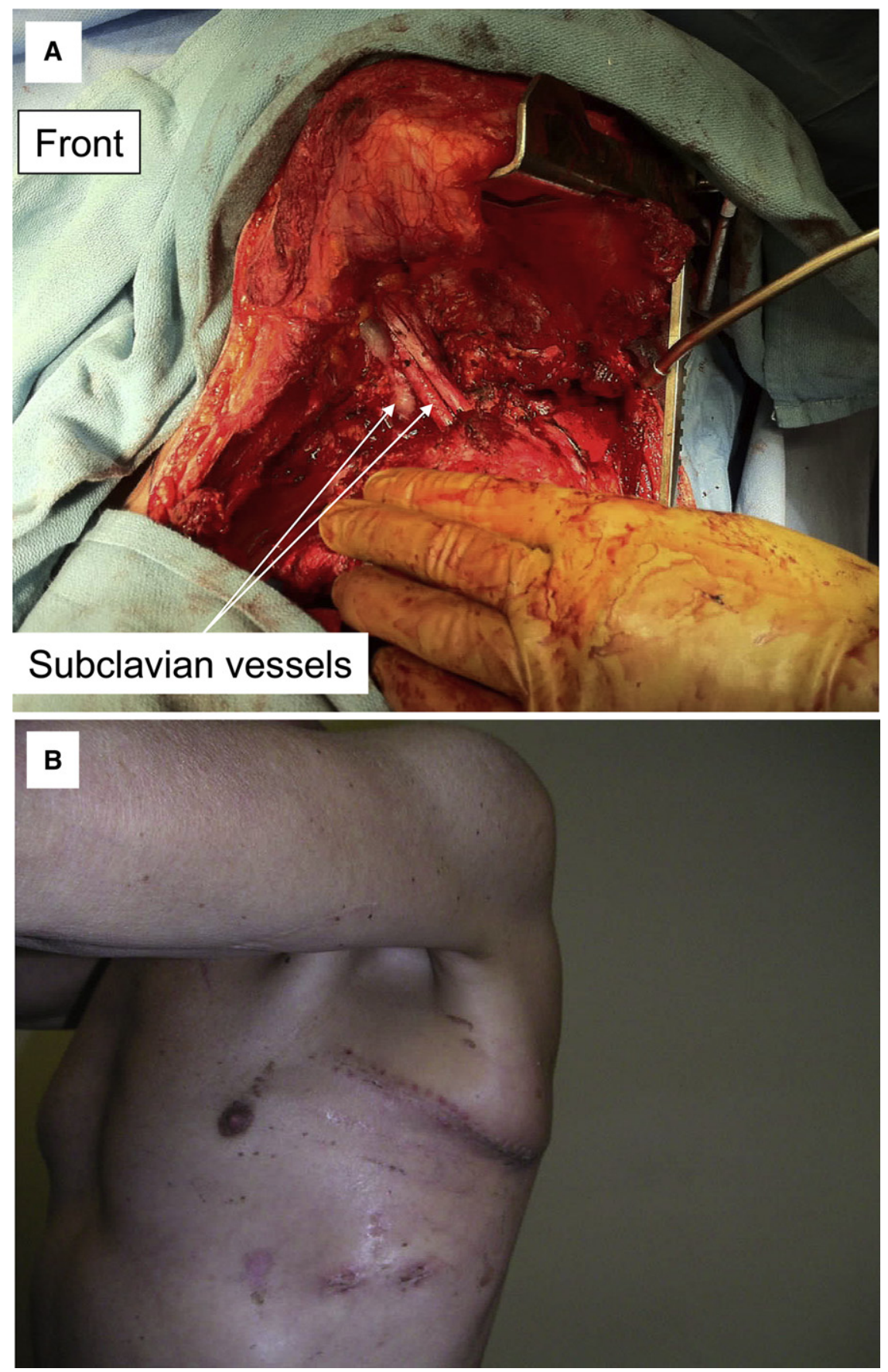

FIGURE 6. A, Intraoperative view and (B) postoperative photograph of the posterotransaxillary approach with an incision extending around the scapula from $\mathrm{C} 7$ posteriorly to the lateral border of the major pectoralis muscle anteriorly. The axillary extension of the incision provided excellent exposure to the subclavian artery distally to the lateral border of the anterior and middle scalene muscles. A and B, The same view from the left side of the patient.

the major pectoralis muscle in the axilla (posterotransaxillary approach) in 1 patient with a posterior tumor extending laterally to the subclavian vessels. The posterolateral approach could also be combined with a long posterior midline incision to perform partial or total vertebrectomy of the spine at the level of $\mathrm{T} 2$ and below, en bloc with lung resection for NSCLC. However, the posterolateral approach provided minimal exposure to the $\mathrm{T} 1$ vertebra, and a transclavicular approach, rather than a posterolateral approach, was used when T1 was involved as described above.

The present study was limited by the small number of patients owing to the rarity of these tumors. Patients with nonmalignant tumors were also excluded because benign diseases are often approach with more limited access. The 
present analysis included only our experience; therefore, the surgical approaches based on the anatomic location of the tumor might vary according to surgeon experience and the degree of comfort with each approach. However, we believe that division of the thoracic inlet into the suggested zones is important because each zone requires specific surgical considerations and will provide guidance for thoracic surgeons in selecting the approach that best enables the surgical goals.

\section{CONCLUSIONS}

A number of surgical approaches have been developed during the past 20 years to access the thoracic inlet. Because no single approach provides the best access for all tumors located in this region, thoracic surgeons must choose the most appropriate incision for each patient. The classic division of the apex of the chest into the anterior, middle, and posterior compartment with an anterior approach for the anterior and middle compartment and a posterior approach for the posterior compartment does not provide enough information to help the surgeon select the most adequate surgical option. The present study reports on 5 zones in the thoracic inlet that require specific surgical considerations and potentially different surgical approaches. The difference between zone 1 and 2 is important, because tumors located in zone 1 will require opening of the costoclavicular space to expose the subclavian vein, and tumors in zone 2 might involve the subclavian artery and brachiocephalic vein, but not the subclavian vein, at the level of the thoracic inlet. In our experience, zone 1 was better accessed by a subclavicular approach to achieve adequate exposure of the subclavian vein, and zone 2 was better accessed by the supraclavicular approach to expose the subclavian artery. The difference between zones 3 and 4 also has important surgical implications. Tumors located in zone 3 typically involve the vertebral artery and/or T1 vertebra and are therefore technically extremely difficult to expose from a posterior approach to achieve an en bloc resection. In our experience, the transclavicular approach was ideally suited to expose this zone, because it provides complete reflection of the sternocleidomastoid muscle with a myocutaneous flap back to the posterior triangle of the neck and thus anterolateral exposure to this zone. Tumors located in zone 5 are less common; however, we believe that transaxillary extension of the posterior approach is particularly helpful to expose tumors located in this zone and should be thus be differentiated from zone 4 .

\section{References}

1. Dartevelle P, Macchiarini P. Surgical management of superior sulcus tumors. Oncologist. 1999;4:398-407.

2. Rusch VW. Management of pancoast tumours. Lancet Oncol. 2006;7:997-1005.

3. Macchiarini P. Resection of superior sulcus carcinomas (anterior approach). Thorac Surg Clin. 2004;14:229-40.

4. Anraku M, Waddell TK, de Perrot M, Lewis SJ, Pierre AF, Darling GE, et al. Induction chemoradiotherapy facilitates radical resection of T4 non-small cell lung cancer invading the spine. J Thorac Cardiovasc Surg. 2009;137:441-7.

5. Blanchereau P, Alric P, Berthet JP, Leger P, Mary H, Marty-Ane C. Surgical exposure of superior sulcus lung tumors with vascular involvement. Ann Vasc Surg. 2001;15:206-11.

6. Hajarizadeh H, Rohrer MJ, Cutler BS. Surgical exposure of the left subclavian artery by median sternotomy and left supraclavicular extension. J Trauma. 1996;41:136-9.

7. Masaoka A, Ito Y, Yasumitsu T. Anterior approach for tumor of the superior sulcus. J Thorac Cardiovasc Surg. 1979;78:413-5.

8. Murphy TO, Piper CA, Kanar EA, McAlexander RA. Subclavicular approach to first rib resection. Am J Surg. 1980;139:634-6.

9. de Perrot M. Resection of superior sulcus tumors: anterior approach. $O p$ Tech Thorac Cardiovasc Surg. 2011;16:138-53.

10. Grunenwald D, Spaggiari L. Transmanubrial osteomuscular sparing approach for apical chest tumors. Ann Thorac Surg. 1997;63:563-6.

11. Ladas G, Rhys-Evans PH, Goldstraw P. Anterior cervical-transsternal approach for resection of benign tumors at the thoracic inlet. Ann Thorac Surg. 1999;67: 785-9.

12. Korst RJ, Burt ME. Cervicothoracic tumors: results of resection by the "hemiclamshell” approach. J Thorac Cardiovasc Surg. 1998;115:286-94.

13. Dartevelle PG, Chapelier AR, Macchiarini P, Lenot B, Cerrina J, Ladurie FL, et al. Anterior transcervical-thoracic approach for radical resection of lung tumors invading the thoracic inlet. J Thorac Cardiovasc Surg. 1993;105: 1025-34.

14. Shaw RR, Paulson DL, Kee JL. Treatment of superior sulcus tumor by irradiation followed by resection. Ann Surg. 1961;154:29-40.

15. Niwa H, Masaoka A, Yamakawa Y, Fukai I, Kiriyama M. Surgical therapy for apical invasive lung cancer: different approaches according to tumor location. Lung Cancer. 1993;10:63-71.

16. Tatsumura T, Sato H, Mori A, Miyazaki M, Koyama S, Tsujimoto M, Furuno T. A new surgical approach to apical segment lung diseases, including carcinomas and inflammatory diseases. J Thorac Cardiovasc Surg. 1994;107:32-6.

17. Nomori H, Nara S, Horio H. Modified trap-door thoracotomy for malignancies invading the subclavian and innominate vessels. Thorac Cardiovasc Surg. 1995;43:204-7.

18. Rusca M, Carbognani P, Bobbio P. The modified "hemiclamshell" approach for tumors of the cervicothoracic junction. Ann Thorac Surg. 2000;69:1961-3.

19. Matsuguma H, Nakahara R, Ishikawa Y, Suzuki H, Ui A, Yokoi K. Transmanubrial osteomuscular sparing approach for lung cancer invading the anterior part of the thoracic inlet. Gen Thorac Cardiovasc Surg. 2010;58:149-54.

20. Fadel E, Missenard G, Chapelier A, Mussot S, Leroy-Ladurie F, Cerrina J, Dartevelle P. En bloc resection of non-small cell lung cancer invading the thoracic inlet and intervertebral foramina. J Thorac Cardiovasc Surg. 2002;123: 676-85.

21. de Perrot M, Rampersaud R. Anterior transclavicular approach to malignant tumors of the thoracic inlet: importance of the scapulothoracic articulation. $J$ Thorac Cardiovasc Surg. 2007;134:801-3.

22. Marshall MB, Kucharczuk JC, Shrager JB, Kaiser LR. Anterior surgical approaches to the thoracic outlet. J Thorac Cardiovasc Surg. 2006;131: 1255-60.

23. Onuki T, Murasugi M, Mae M, Sone Y, Kei J, Nitta S. Modification of anterior approach to superior sulcus tumors. J Thorac Cardiovasc Surg. 1998;116:663-4.

24. Birch R, Bonney G, Marshall RW. A surgical approach to the cervicothoracic spine. J Bone Joint Surg. 1990;72:904-7. 